\title{
Nematics with Quenched Disorder: Pinning out the Origin of Memory
}

\author{
M. Rotunno, ${ }^{1}$ M. Buscaglia, ${ }^{1}$ C. Chiccoli, ${ }^{2}$ F. Mantegazza, ${ }^{3}$ P. Pasini, ${ }^{2}$ T. Bellini, ${ }^{1}$ and C. Zannoni ${ }^{4}$ \\ ${ }^{1}$ Dipartimento di Chimica, Biochimica e Biotecnologie per la Medicina and INFM, Università di Milano, Italy \\ ${ }^{2}$ Istituto Nazionale di Fisica Nucleare, Sezione di Bologna, Via Irnerio 46, 40126 Bologna, Italy \\ ${ }^{3}$ INFM and DIMESAB, Università di Milano-Bicocca, Via Cadore 48, 20052 Monza (MI), Italy \\ ${ }^{4}$ Dipartimento di Chimica Fisica and INSTM, Università di Bologna, Viale Risorgimento 4, 40136 Bologna, Italy
}

(Received 3 September 2004; published 11 March 2005)

\begin{abstract}
Memory effects and glassy behavior have been repeatedly observed in disordered nematic liquid crystals but the connection between these effects and the system topology remained unrevealed. We present an analysis of the local and global topology of the nematic ordering in the presence of quenched disorder and we show that nematics with quenched disorder can be mapped into a system of pinned defect lines and that the memory of the system stems from the pinning of these strings.
\end{abstract}

DOI: 10.1103/PhysRevLett.94.097802

One of the major revolutions in condensed matter physics is the shift from depicting defects as imperfections of an otherwise ordered matrix to considering them as entities characterized by topological charge, mutual interactions, and ordering transitions [1]. This change of focus has enabled reinterpreting classical order-disorder transitions (in solids, superconductors, superfluids, and liquid crystals) in terms of defects, e.g., disordering transitions as processes of defect proliferation and unbinding. Although this has been most intensively investigated in type-II superconductors, where vortex lines have been found to offer an amazing potential for phase variety [2,3], quite similar, but perhaps even richer, situations can be found in liquid crystals (LCs) [4,5]. Indeed, the smectic LC phases, as also expected on the basis of the famous analogy with superconductors, hosts a rich variety of defect structures $[1,6]$. In this same vein the nematic $(N)$ to isotropic $(I)$ phase transition of LCs reflects the blowout (or condensation) of disclination defect lines (DL) $[7,8]$. Computer simulations have demonstrated that by changing the defect topology it is possible to make the transition more-or less-pronounced [4,9]. We have previous shown that the behavior of nematics hosting random solid networks can be successfully described by the sprinkled silica spin (SSS) model $[10,11]$, an idealized spin lattice with static random disorder. Experiments and SSS simulations coherently confirmed the short ranged nature of local ordering [10] and the presence of disorder induced memory effects [11]. Here we present an analysis of SSS simulation results for the local and global topology of $N$ order in the presence of quenched disorder and we show for the first time that nematics with quenched disorder can be mapped into a system of pinned defect lines and that the dependence of the system properties on its thermal history is a consequence of the pinning of long disclination DL by quenched disorder. The thermal history of the samples determines the population of DL, while the pinning strength marks the threshold from elastic response to memory effects. This scenario presents indeed many intriguing analogies with the description of vortex glasses in disordered type-II
PACS numbers: 61.30.Jf, 61.30.Cz

superconductors [12], also studied by computer simulations $[13,14]$.

Two basic forms of memory are found in $N$ with quenched disorder: field-cooling (FC) memory, already reported in a previous work ([11], Fig. 3), and permanence of partial alignment in zero-FC (ZFC) samples when temporarily subjected to external electric fields. Quite likely both forms of memory play a role in determining the possibility of permanent "writing" of "filled nematics" displays [15]. Both forms of memory are canceled when samples are brought in the $I$ phase, although for very high fields (a situation not explored here) permanent restructuring of the disordering network or of LC anchoring at the solid interface can take place [16]. The field-induced memory on ZFC samples, typically requiring larger fields than the FC memory, is reported in Fig. 1(a) for two different species of experimental samples (Filled nematics and Millipore-LCs). In turn, at low fields, where the permanent field-induced ordering is negligible, the whole system behavior is elastic [17]. The structural difference between the two disordering matrices and the arguable flexibility of the Aerosil branches may account for the diversity in field threshold and in residual transmittance.

The SSS model [10] is a Lebwohl-Lasher model with quenched disorder, defined by the following Hamiltonian:

$$
H_{\mathrm{SSS}}=-\epsilon\left[\sum_{\langle i, j\rangle \in(\mathcal{N} \cup S)} P_{2}\left(\mathbf{s}_{i} \cdot \mathbf{s}_{j}\right)+\eta \sum_{i \in \mathcal{N}} P_{2}\left(\mathbf{s}_{i} \cdot \mathbf{z}\right)\right]
$$

where $i, j$ run on nearest neighbors $i<j$ and a fraction $p$ of the spins $\left(\mathbf{s}_{i}\right)$, randomly chosen, is kept fixed in random orientations. Thus the system is composed by the two sets: $\mathcal{N}$-free spin describing the LC-frozen and $S$-frozen spins (FS) introducing quenched disorder in the system, mimicking, for example, silica inclusions. The positive constants $\epsilon, \eta$ express the spin-spin and field-spin coupling. Here the field is along $Z$ and $P_{2}$ is the 2nd Legendre polynomial. The evolution of the system is studied, using the Monte Carlo (MC) METROPOLIS algorithm [18], on cubic lattice systems of linear sizes $L=50,80$, and with 


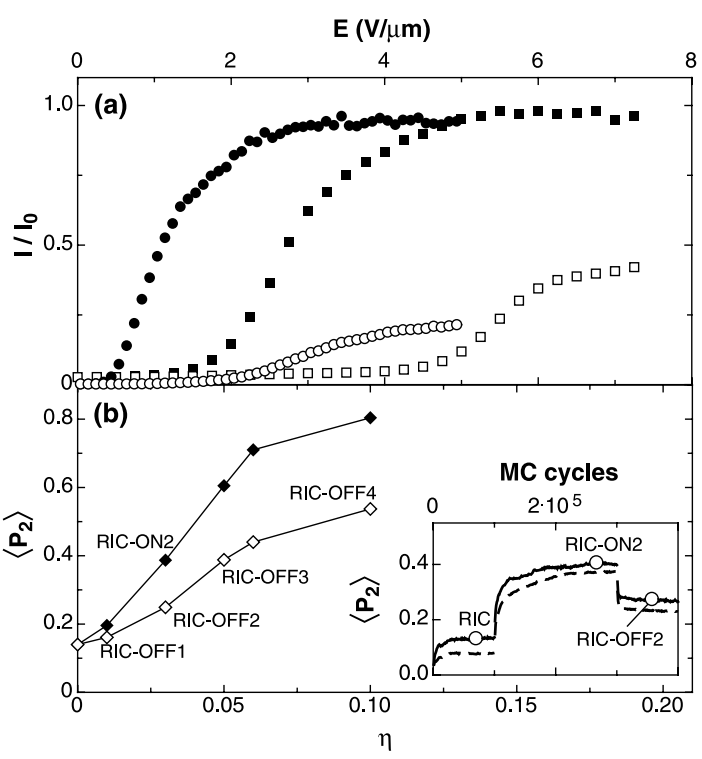

FIG. 1. Comparison between data obtained by optical measurements [Panel (a)] and SSS MC simulations [Panel (b)] in a ZFC experiment: the system is quenched in the $N$ phase with no external electric field applied. After the equilibration an electric pulse is applied and the data are collected in presence (filled symbols) and after the removal (open symbols) of the applied field $E$. Panel (a) Transmitted intensity normalized to the isotropic value $I / I_{0}$ measured as a function of the amplitude of $1 \mathrm{kHz}$ electric field applied for 5 seconds. The samples are thermalized at $10^{\circ} \mathrm{C}$ below the $I$ to $N$ transition temperature. Circles: $3 \mu \mathrm{m}$ Millipore membrane imbibed by $5 \mathrm{CB}$ [11]. Squares: Filled nematic composed by $6 \mathrm{CB}$ and $15 \%$ Aerosil [10]. Panel (b) Asymptotic order parameter $\left\langle P_{2}\right\rangle$ plotted as a function of electric field strength $\eta$. Data obtained with a $50^{3}$ cube with $p=14 \%$. Inset: $\left\langle P_{2}\right\rangle$ plotted as a function of the MC cycles for $L=50$ (solid line) and $L=80$ (dashed line). The electric field $\eta=0.03$ is $\mathrm{ON}$ in the MC cycles interval $[100000,200000]$ and OFF elsewhere. In the inset three states are defined: the initial state (zero-FC or RIC), the ON asymptotic state in the presence of a field, and the OFF asymptotic state after the removal of the field. The acronyms in the figures indicate the configurations described in the text and in Table I.

$p=0.07,0.14$. Typically we show results after $10^{5}$ cycles, a $\mathrm{MC}$ time more than sufficient to reach equilibrium for normal $N$ phases [18]. The value of $\mathrm{P} 2$ for these equilibrated states is generally overestimated because of finitesize effect and decreases with increasing lattice size [12] as shown in the inset of Fig. 1. In the simulations we mimic the FC and ZFC states by studying the evolution from an aligned initial configuration ( $\mathrm{AIC}_{0}$, with all aligned spins) and a random initial configuration $\left(\mathrm{RIC}_{0}\right)$ [11]. The corresponding equilibrated configurations, here after referred to as AIC and RIC states, respectively, are remarkably different [11]. In Fig. 1(b) we show, in analogy to the experiments in Fig. 1(a), the presence of field-induced memory in zero-FC states. The remarkable analogy in the memory effects found in experiments and simulations indicates that such effects have a universal character going beyond the chemical details of the mesogen.
Our simulations aimed to unravel the basic differences between the various equilibrium (or metastable) states: FC, ZFC, and ZFC-OFF states (defined in Fig. 1). A crucial tool for this analysis was locating the disclination lines, stable DL in the $N$ phase, their annihilation being possible only through the collapse of defect loops. We use a method mathematically equivalent to that of Zapotocky et al. [19], and consider all the elementary squares formed in the simulation lattice by neighboring spins $\mathbf{s}_{i}, \mathbf{s}_{j}, \mathbf{s}_{k}, \mathbf{s}_{l}$. A defect segment pierces the square when the sign of the product $\prod_{4}=\left(\mathbf{s}_{i} \cdot \mathbf{s}_{j}\right)\left(\mathbf{s}_{j} \cdot \mathbf{s}_{k}\right)\left(\mathbf{s}_{k} \cdot \mathbf{s}_{l}\right)\left(\mathbf{s}_{l} \cdot \mathbf{s}_{i}\right)$ is negative. (see Ref. [20]). With this algorithm we detected a large number of DL, all forming loops of various lengths. The analysis of the number and extension of defects in equilibrated systems, shown in Fig. 2 and in Table I, reveals a remarkable difference between AIC vs RIC states. The AIC states contain a slightly shorter total DL length with DLs organized in short loops $(<100)$, while the DLs of RIC states, whose distribution in perimeter length approaches the AIC one for short DLs, also feature some very extended loops $(>100)$, absent in the AIC states. A snapshot of the longest defect line in the $L=50, p=0.14$ RIC state is shown in the inset of Fig. 2 (blue line).

Analysis of perimeter and persistence lengths of long DL reveals their intimate connection with the structuring of the system on length scales larger than the nematic correlation length $\zeta$. To this aim we performed a new set of simulations as follows: in both AIC and RIC states, we have frozen all spins involved in regions of large local ordering (i.e,. spins whose energy is lower than a given threshold) and found a new stable state freeing all other spins, previous FS included. The energy thresholds here employed were -2.75 for $p=0.07$ and -2.65 for $p=0.14$ and

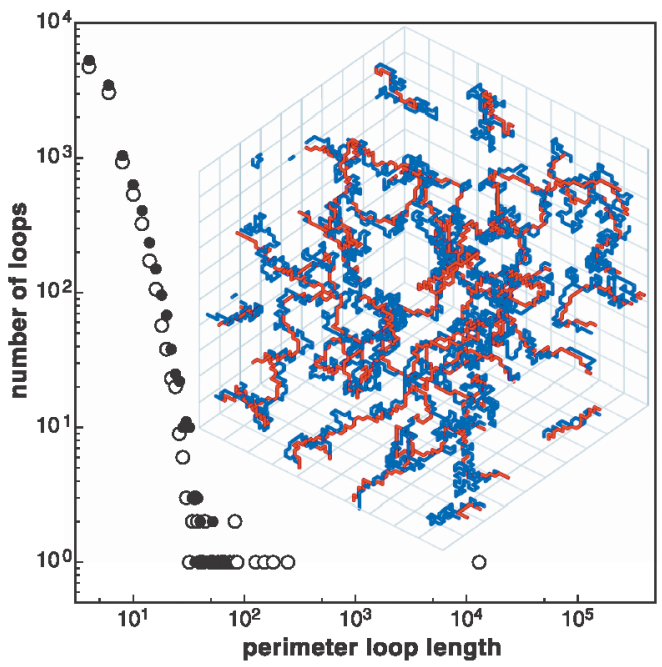

FIG. 2 (color). DL perimeter length distribution for AIC (dots) and RIC (open circles) for $L=80$ and $p=0.14$. Inset: snapshot of the longest DL (2968 lattice units) in a RIC state, $p=0.14$, $L=50$ (blue line). The red line is the longest DL (1392 lattice units) obtained through the domain freezing procedure applied to the same RIC system (see text for details). 
TABLE I. DL parameters in the various states of the MC simulations with disorder density $(p)$, lattice size $(L)$ and for the distribution of constraints. The first line of the table represents an equilibrated state with no frozen spins. For the other states, the random FS are the same for all the states, except the FD states, where the $p$ value, labeled as " **", refers to the RIC state whose ordered domains have been frozen (see text for details). The longest DL loop length $\left(L_{\mathrm{max}}^{\mathrm{DL}}\right)$ marks the main topological difference between AIC, RIC, and RIC-OFF states: the macroscopic remnant order $\left\langle P_{2}\right\rangle$ in AIC and RIC-OFF states couples to a remarkable $L_{\text {max }}^{\mathrm{DL}}$ reduction. The energy per spin $U$ for a given $p$ is system history independent. The $N$ correlation length $\zeta$, expressing the extension of the local $N$ ordering, is well matched by the persistence distance $d_{p}$, defined as the distance associated to the persistence length $L_{p}$ (see text for details). Reported data are averages on equilibrated configurations, with a standard deviation $\approx 5 \%$.

\begin{tabular}{lllrrrrr}
\hline \hline \multicolumn{1}{c}{ state } & \multicolumn{1}{c}{$L$} & \multicolumn{1}{c}{$L_{\text {tot }}^{\mathrm{DL}}$} & $L_{\max }^{\mathrm{DL}}$ & $U$ & $\left\langle P_{2}\right\rangle$ & $\zeta$ \\
\hline$\ldots$ & 0 & 30 & 0 & 0 & -2.79 & 0.95 & \\
AIC & 0.07 & 80 & 29040 & 30 & -2.58 & 0.58 & \\
RIC & 0.07 & 80 & 30500 & 3700 & -2.57 & 0.18 & 10.4 \\
RIC-FD & $0.07 *$ & 80 & 2600 & 2400 & -2.70 & 0.19 & 10.4 \\
AIC & 0.14 & 80 & 74000 & 60 & -2.38 & 0.45 & $18(10.2)$ \\
RIC & 0.14 & 80 & 76000 & 12400 & -2.36 & 0.07 & 6.0 \\
RIC-FD & $0.14 *$ & 80 & 6500 & 3700 & -2.59 & 0.08 & 6.0 \\
AIC & 0.14 & 50 & 18200 & 50 & -2.38 & 0.56 & \\
RIC & 0.14 & 50 & 18280 & 2970 & -2.36 & 0.13 & \\
RIC-OFF1 & 0.14 & 50 & 18260 & 2490 & -2.36 & 0.16 & \\
RIC-OFF2 & 0.14 & 50 & 18220 & 2210 & -2.37 & 0.25 & \\
RIC-OFF3 & 0.14 & 50 & 18140 & 1470 & -2.37 & 0.39 & \\
RIC-OFF4 & 0.14 & 50 & 18300 & 50 & -2.38 & 0.56 & \\
\hline \hline
\end{tabular}

implied freezing, respectively, $82 \%$ and $73 \%$ of the spin number. While in the case of AIC this "domain freezing" procedure yields a totally defect-free state, in the case of RIC it yields a state having only long DL with basic trajectory similar to those of the original RIC state but definitely smoother (red line in the inset of Fig. 2). We find that the shape of the correlation function $g_{N}(r)$ for the $N$ order does not change after the domain freezing procedure (see the value of $\zeta$ in Table I). This matching demonstrates that the presence of a long DL running a specific path is essential to the RIC structure on long length scale. The very necessity of long DL follows from the notion of short ranged order, as can be seen by idealizing the system as a collection of uncorrelated domains of size $\zeta$. For three randomly oriented neighboring domains there is a probability of $32 \%$ that the product $\prod_{3}$ calculated on their orientation is negative and consequently that a DL runs across the ideal triangle joining the domains. By adopting the simplest assumption that domains are arranged in a regular close packing geometry, we can estimate the number of such triangles and thus the total DL length. We obtain $L_{\mathrm{tot}}^{\mathrm{DL}} \approx 2400(7100)$ for $p=0.07(0.14)$ and $L=$ 80 , remarkably close to the total length of DL in RIC "domain freezing" simulations.

The intimate relationship between DL and correlation length becomes apparent considering the persistence length $L_{p}$ of the long DL loops, defined as the characteristic length of the exponential decay of the DL orientation correlation $f_{p}(l)=\langle\mathbf{u}(i) \cdot \mathbf{u}(i+l)\rangle$, where $\mathbf{u}$ is a unit vector oriented as each defect segment. The values of $L_{p}$ are shown in Table I for both RIC and RIC "frozen domain"(FD) states. Since $L_{p}$ refers to a contour length, to be compared with $\zeta$ it needs to be converted into the corresponding "persistence distance" $d_{p}$ (shown in Table I in parenthesis for each $L_{p}$ value), i.e., the average distance between two points having a contour length $L_{p}$ on a DL. Equal values of $d_{p}$ are found for RIC and RIC-FD, despite the difference in $L_{p}$. In all considered cases the values of $\zeta$ and $d_{p}$ remarkably match.

According to this analysis, memory effects in the orientational order of random nematics mirror memory effects in the topological state of the system. In turn, the presence of DL has to be ascribed to the quenched disorder, as it evidently appears by considering the ratio $R=$ (number of $\mathrm{DL}$ neighboring at least one $\mathrm{FS}$ )/ (total DL number) $>0.96$ always (in RIC, RIC-ON, RIC-OFF, and AIC states, with $p=0.07$ and 0.14). This value should be compared with what is expected if the DL were randomly coupled to the FS, i.e., the ratio (elementary squares containing at least one FS)/ (total number of elementary squares) $=0.45(p=0.14)$ and $0.25(p=0.07)$. DL, both long and short, are thus "attracted" by FS. To confirm this notion, we inspected the field effects on DL, since the application of an external field enables us to permanently modify the ordering, unaffected by thermal motion. The results in Table I show that the RIC-OFF1-4 states (defined in Fig. 1), have increasing $\left\langle P_{2}\right\rangle$ and decreasing $L_{\max }^{\mathrm{DL}}$, whereas both their total DL length and energy are basically constant. The typical pattern by which the long DL shrinks as the field is applied, is exemplified by the snapshot in Fig. 3(b). As apparent, field application changes portions of DL only, while relevant parts of them keep track of the original RIC DL. In order to quantify the shrinking, splitting, and pinning of DL qualitatively shown in Fig. 3(b), we have defined a proximity parameter (PP) [see stars in Fig. 3(a)] which compares the 


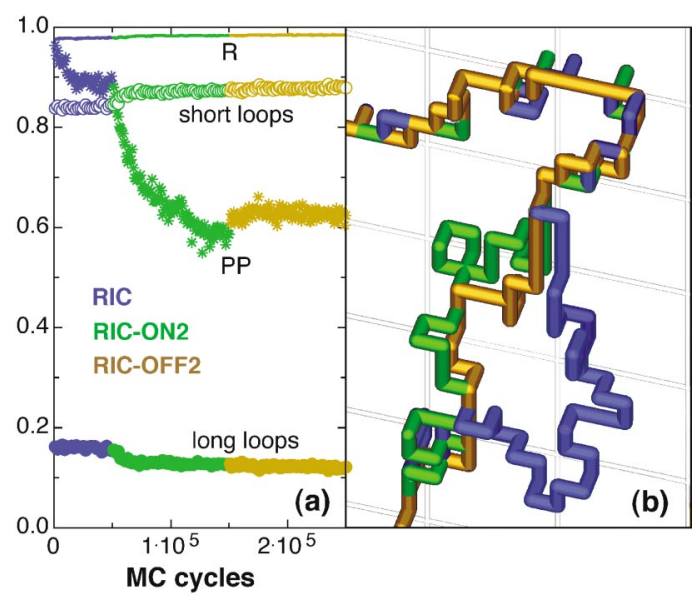

FIG. 3 (color). Panel (a) Evolution of the relative fraction of long (open circles) and short (filled circles) defect loops, PP (stars), and $R$ fraction (lines) as a function of MC cycles. The data refer to the same MC simulations presented in the inset of Fig. 1(b). The blue, green, and orange symbols refer to states where the field is OFF, ON, and OFF again, respectively. Panel (b) Snapshot of DL in a small portion of the simulation cube, comparing the RIC state (blue line), the RIC-ON2 state (green line) and RIC-OFF2 state (orange line).

trajectory of the long DL of a specific RIC configuration with its subsequent evolution. PP is defined as the fraction of the DL having a distance $\leq \sqrt{2}$ lattice units from the original RIC DL, thus classifying as "near" DL belonging to the same elementary cube. The effects of the field application and of its removal are shown in Fig. 3(a), which refers to the same data shown in the inset of Fig. 1(b). We find that indeed a large fraction of the DL remains bound to its original path, and that as the field is removed, the PP displays a slight increase, indicating that the DLs partially go back to their original path. Also shown in Fig. 3(a) are the parameter $R$ (line) and the relative fraction of $\mathrm{DL}$ belonging to short $(<100$, open dots $)$ and long $(>100$, full dots) loops. The combination of evidences offered by Fig. 3 indicates that FS bind DL through specific "pinning" sites, favoring the positioning of DL in definite locations. As the field is applied, long DL are stretched away from the weakest pinning sections (PP decreases) and partly broken into short DL loops, as indicated by the decrease (increase) of the number of DL belonging to long (short) loops. Since these transformations occur at constant $R$, turning off the field produces only small adjustments in the DL, which are captured by their closest pinning sites formed by FS. Accordingly, the various configurations, from the RIC to the AIC through the RIC-OFF states, all equivalent in energy, appear as separated by energy barriers related to the depinning and the splitting of DL. Ultimately, the notion of FS-induced pinning sites is implied by the fact that the total DL number is the same in RIC and AIC states. Although it has not yet been demonstrated, we argue that the random nature of the FS implies they cannot be smoothly connected without DL. Thus, local energy minimization certainly favors specific locations for the DL, which can be satisfied by either short or long DL loops.

In summary we have shown that the multitude of stable configurations observed in experiments and computer simulations of nematics with quenched disorder correspond to radically different topological states, characterized by different distributions of long and short DL, and separated by energy barriers corresponding to DL depinning and splitting events. This work sheds a new light on the intimate connection between quenched disorder and glassy behavior [3]. The analogy between LCs and superconductors normally goes through the more complex smectic phases [6], rather than the nematic studied here. The impressive effect of coupling defect lines to quenched disorder seems to establish a new correspondence between the two fields, suggestive of a more fundamental pattern in disordered materials.

C. Z. thanks MIUR (COFIN "Cristalli Liquidi") and EU. T. B. thanks MIUR (COFIN 2004024508).

[1] P. M. Chaikin and T.C. Lubensky, Principles of Condensed Matter Physics (Cambridge University Press, Cambridge, 1995).

[2] D. S. Fisher, M. P. A. Fisher, and D. A. Huse, Phys. Rev. B 43, 130 (1991).

[3] D. R. Nelson, Nature (London) 430, 840 (2004).

[4] M. J. Bowick et al., Science 263, 943 (1994).

[5] Defects in Liquid Crystals: Computer Simulations, Theory and Experiments, edited by O. D. Lavrentovich, P. Pasini, C. Zannoni, and S. Žumer (Kluwer, Dordrecht, 2001).

[6] P. G. de Gennes, Solid State Commun. 10, 753 (1972).

[7] J. Toner, P. E. Lammert, and D. S. Rokhsar, Phys. Rev. E 52, 1801 (1995).

[8] N. V. Priezjev and R. A. Pelcovits, Phys. Rev. E 64, 031710 (2001).

[9] P.E. Lammert, D.S. Rokhsar, and J. Toner, Phys. Rev. Lett. 70, 1650 (1993).

[10] T. Bellini et al., Phys. Rev. Lett. 85, 1008 (2000).

[11] T. Bellini et al., Phys. Rev. Lett. 88, 245506 (2002).

[12] D. A. Huse, M.P. A. Fisher, and D. S. Fisher, Nature (London) 358, 553 (1992).

[13] A. Vestergren, J. Lidmar, and M. Wallin, Phys. Rev. Lett. 88, 117004 (2002).

[14] P. Olsson, Phys. Rev. Lett. 91, 077002 (2003).

[15] M. Kreuzer and R. Eidenschink in Liquid Crystals in Complex Geometries, edited by G.P. Crawford and S. Žumer, (Taylor \& Francis, London, 1996).

[16] D. Liang, M. A. Borthwick, and R. L. Leheny, J. Phys. Condens. Matter 16, S1989 (2004).

[17] M. Buscaglia et al., Europhys. Lett. 48, 634 (1999).

[18] Advances in the Computer Simulation of Liquid Crystals, edited by P. Pasini and C. Zannoni (Kluwer, Dordrecht, 2000).

[19] M. Zapotocky, P. M. Goldbart, and N. Goldenfeld, Phys. Rev. E 51, 1216 (1995).

[20] P. E. Lammert, D. S.Rokhsar, and J. Toner, Phys. Rev. E, 52, 1778 (1995). 